\title{
ARTICLE
}

Received 18 Jun 2013 | Accepted 22 Oct 2013 | Published 18 Nov $2013 \quad$ DOl: 10.1038/ncomms3805

\section{Orai1-dependent calcium entry promotes skeletal muscle growth and limits fatigue}

Lan Wei-LaPierre ${ }^{1}$, Ellie M. Carrell ${ }^{1}$, Simona Boncompagni ${ }^{2}$, Feliciano Protasi $^{2}$ \& Robert T. Dirksen ${ }^{1}$

Store-operated $\mathrm{Ca}^{2+}$ entry (SOCE) in skeletal muscle involves signalling between stromalinteracting molecule 1 (STIM1) in the sarcoplasmic reticulum (SR) and $\mathrm{Ca}^{2}+$ selective Orai1 channels in the sarcolemma. Here we generate transgenic mice with muscle-specific expression of dominant-negative Orai1 (dnOrai1) and demonstrate that Orai1-dependent SOCE promotes growth and limits fatigue in adult skeletal muscle. dnOrai1 mice lack SOCE specifically in muscle but are fertile and thrive well into adulthood. Although muscle ultrastructure, excitation-contraction (EC) coupling, fibre type, and expression of other $\mathrm{Ca}^{2+}$ regulatory proteins are unaltered, dnOrai1 mice exhibit reduced body weight, muscle mass and fibre cross-sectional area. Importantly, during intense repetitive activity, dnOrai1 mice display increased susceptibility to fatigue at the single fibre, excised muscle and whole-animal levels. We further show that STIM1 and Orai1 proteins co-localize within the triad junction but do not exist in a preassembled context. These results show that Orai1-dependent SOCE has an important physiological role in muscles of adult mice.

\footnotetext{
${ }^{1}$ Department of Physiology and Pharmacology, University of Rochester, 601 Elmwood Avenue, Rochester, New York 14642, USA. ${ }^{2}$ CeSI-Center for Research on Ageing \& DNI-Department of Neuroscience and Imaging, University G. d'Annunzio, Chieti I-66013, Italy. Correspondence and requests for materials should be addressed to R.T.D. (email: Robert_Dirksen@URMC.Rochester.edu).
} 
$\mathrm{S}$ tore-operated $\mathrm{Ca}^{2+}$ entry (SOCE) is activated by depletion of intracellular $\mathrm{Ca}^{2+}$ stores $^{1}$. In non-excitable cells, SOCE is coordinated by stromal-interacting molecule 1 (STIM1) serving as an endoplasmic reticulum (ER) $\mathrm{Ca}^{2+}$ sensor $^{2-4}$ that activates $\mathrm{Ca}^{2+}$-selective Orail channels in the plasma membrane $e^{5,6}$. Specifically, store depletion results in $\mathrm{Ca}^{2+}$ unbinding from the luminal EF hand (helix-loop-helix) motif of STIM1, which induces STIM1 aggregation and translocation to adjacent ER plasma membrane junctions. Within these junctions, STIM1 aggregates interact with Orail to activate the channel and promote $\mathrm{Ca}^{2}+$ influx ${ }^{7-10}$.

SOCE in skeletal myotubes exhibits a similar biophysical, pharmacological and molecular signature as that observed in non-excitable cells. SOCE in myotubes is activated by depletion of $\mathrm{SR} \mathrm{Ca}^{2+}$ stores and is inhibited by multiple SOCE inhibitors including lanthanide, BTP-2, 2-APB and SK\&F-96365 (refs 11-14). SOCE in myotubes depends on STIM1-Orail coupling as it is abolished by both STIM1 knockdown and expression of human dominant-negative Orail (dnOrail; E106Q) ${ }^{12,14,15}$. SOCE is also absent in myotubes derived from mice with global $^{13}$ or skeletal muscle-specific ${ }^{11}$ deletion of STIM1. Finally, voltage clamp studies ${ }^{14}$ indicate that the SOCE channel current in myotubes exhibits similar biophysical, pharmacological and molecular properties as that observed in $\mathrm{T}$ lymphocytes ${ }^{16,17}$.

Although SOCE in muscle has been linked to $\mathrm{Ca}^{2+}$ store repletion $^{18}$, fatigue ${ }^{19,20}$, NFAT transactivation ${ }^{13,21}$ and muscle differentiation ${ }^{11,13,22}$, the precise physiological role(s) of STIM1Orail coupling and Orail-dependent $\mathrm{Ca}^{2+}$ entry in adult skeletal muscle remains unclear. Rosenberg et al. ${ }^{21}$ reported that global and muscle-specific STIM1 ablation results in a profound reduction in muscle mass, a severe myopathy and neonatal lethality ${ }^{11,13}$. However, STIM1 is a multipurpose stress transducer activated by diverse stimuli (depletion, oxidation, temperature, hypoxia and acidification) that regulates multiple targets including channels (Orail, TrpC, ARC and Cav1.2), pumps/ exchangers (Sarco-Endoplasmic $\mathrm{Ca}^{2}+$ ATPase (SERCA), Plasma membrane $\mathrm{Ca}^{2}+$ ATPase (PMCA), $\mathrm{Na} / \mathrm{Ca}$ exchanger), adaptor proteins (POST), ER chaperones (calnexin and ERp57), signalling enzymes (adenylate cyclase isoform 8) and ER stress/remodelling proteins (microtubule protein EB1) ${ }^{23}$. Thus, the specific role of STIM1-Orail coupling in the survival and complex muscle phenotypes of STIM1 knockout mice is unclear.

To determine the role of Orail-dependent $\mathrm{Ca}^{2+}$ entry in skeletal muscle structure and function, we generated transgenic mice with muscle-specific expression of dnOrail mice. Remarkably, unlike STIM1 knockout mice, dnOrail mice are viable, fertile and live a normal lifespan. Thus, dnOrail transgenic mice enabled determination of the specific role of Orail-dependent $\mathrm{Ca}^{2+}$ entry in skeletal muscle growth, development and performance in adult animals.

\section{Results}

STIM1 and Orail do not form preassembled triad complexes. Although STIM1-Orail coupling underlies SOCE in myotubes, this has not been rigorously determined in fully differentiated adult skeletal muscle fibres. Using immunocytochemical studies in single mouse flexor digitorum brevis (FDB) fibres from adult mice, Orail exhibited a double-row pattern of fluorescence that co-localized with the ryanodine receptor (Fig. 1a), confirming the presence of Orail within the triad junction. STIM1 fluorescence was observed throughout the I-band region, flanking discrete Z-disk localization $\alpha$-actinin, although also significantly enriched with RyR1 in the triad (Fig. 1b,c). Co-localization of STIM1 and Orail in the triad of resting muscle fibres is consistent with a uniquely extremely rapid (for example, tens of milliseconds) and efficient SOCE activation on SR $\mathrm{Ca}^{2}+$ store depletion in muscle ${ }^{14,24-26}$. To support such rapid activation, STIM1 and Orail have been proposed to be closely associated or even form preassembled complexes that are directly activated on store depletion $^{25,27}$. This hypothesis was tested using bimolecular
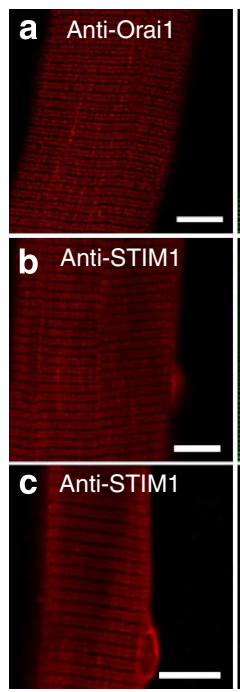
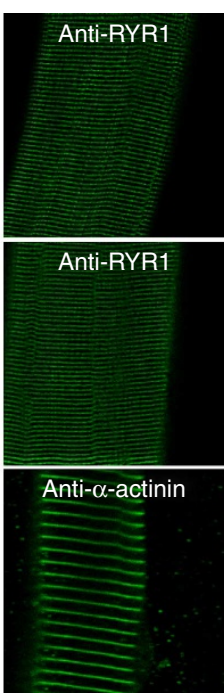

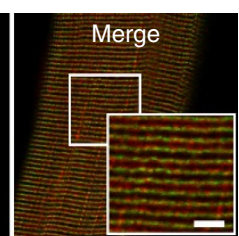

d
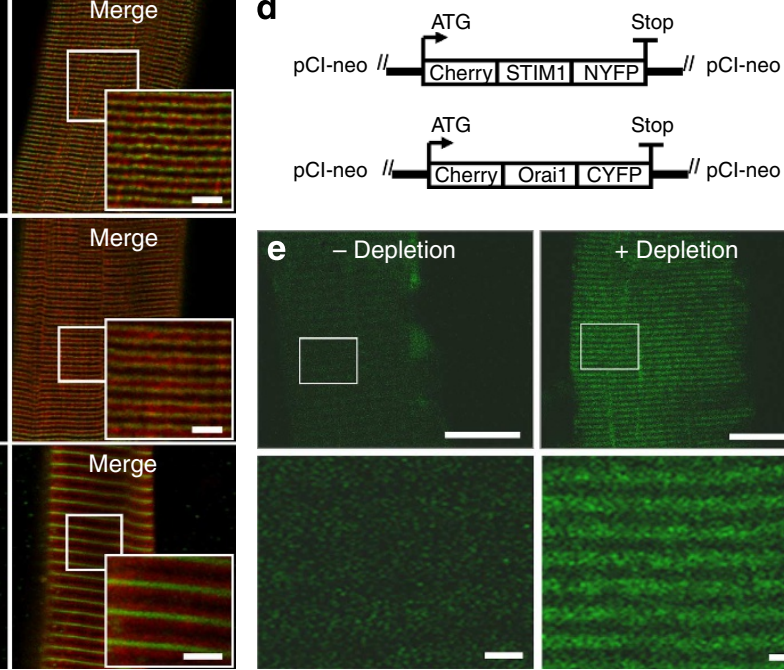
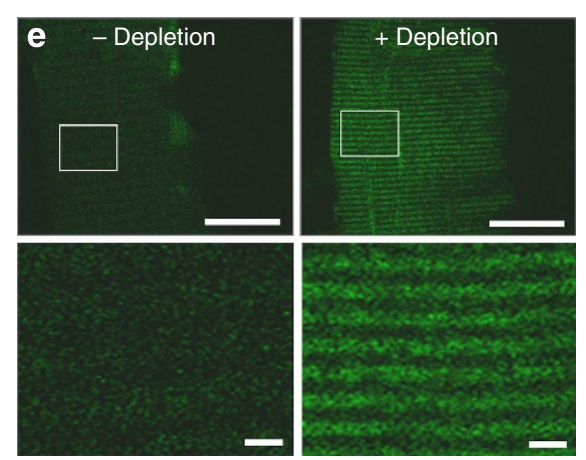

Figure 1 | Co-localization of endogenous STIM1 and Orai1 at the triad junction and formation of STIM1-Orai1 complexes on store depletion.

(a) Representative immunofluorescence images of endogenous Orai1 alone (left), RyR1 alone (middle) and overlay (right) in a FDB fibre from an adult WT mouse. (b) Representative immunofluorescence images of endogenous STIM1 alone (left), RyR1 alone (middle), and overlay (right) in a FDB fibre from an adult WT mouse. (c) Representative immunofluorescence images of endogenous STIM1 alone (left), $\alpha$-actinin alone (middle) and overlay (right) in a FDB fibre from an adult WT mouse. Scale bars, $10 \mu \mathrm{m}$ for low-magnification images and $2 \mu \mathrm{m}$ for high-magnification images. (d) Schematic maps of split YFP constructs used in BiFC experiments. The amino-terminal fragment of YFP (1-158) was fused to the C terminus of cherry-tagged STIM1 (top) and the C-terminal YFP fragment (159-238) was fused to the C terminus of cherry-tagged Orai1 (bottom). (e) Top: representative images of YFP fluorescence in FDB fibres expressing cherry-STIM1-NYFP and cherry-Orai1-CYFP before (left) after (right) TG-induced store depletion. Scale bars, 10 $\mu$ m. (Bottom) Higher-magnification images in the boxed regions shown in the top panels. Scale bars, $2 \mu \mathrm{m}$. 
fluorescence complementation (BiFC) $)^{28-30}$ to determine whether junctional STIM1 and Orail proteins form preassembled SOCE channel complexes in fibres with fully replete $\mathrm{SR} \mathrm{Ca}^{2}+$ stores. FDB muscles were electroporated with complementary DNAs encoding cherry-STIM1-N ${ }_{(1-158)}$ YFP and cherry-Orai1- $\mathrm{C}_{(159-283)}$ YFP. Although expressing fibres lacked basal yellow fluorescent protein (YFP) fluorescence, robust junctional YFP fluorescence was observed following thapsigargin (TG)-induced store depletion (Fig. 1d,e). Thus, although STIM1 and Orail colocalize in the triad, they do not form preassembled STIM1-Orai1 complexes in the absence of SR store depletion.

Characterization of SOCE in adult skeletal muscle fibres. We used $\mathrm{Mn}^{2+}$ quench of fura-2 fluorescence ${ }^{12}$ to examine the molecular determinants and pharmacological signature of SOCE in FDB fibres from adult mice. Although fura-2 emission was unaltered during application of extracellular $\mathrm{Mn}^{2+}$ in nondepleted fibres (Fig. 2a), a significant increase in fura-2 quench rate was observed in TG-treated fibres (Fig. 2b), consistent with robust SOCE activation. $\mathrm{Mn}^{2+}$ quench in store-depleted fibres was inhibited by addition of either $0.2 \mathrm{mM} \mathrm{LaCl}_{3}$ or $10 \mu \mathrm{M}$ BTP-2 (Fig. 2c,d). To assess the importance of Orail in this storedependent entry, cherry-tagged mouse dnOrai1E108Q was transiently expressed by electroporation in FDB muscles of adult mice. TG-pretreated, E108Q-expressing fibres lacked a significant change in fura-2 quench on addition of extracellular $\mathrm{Mn}^{2+}$ (Fig. 2e). Electroporation of FDB muscles with a mixture of four STIM1-specific short-interfering RNAs (siRNAs), but not negative control siRNAs (Supplementary Table S1), markedly reduced expression of both short (average \pm s.e.m.: $62 \pm 12 \%$ ) and long (average \pm s.e.m.: $80 \pm 5 \%$ ) STIM1 isoforms (Supplementary Fig. S1). STIM1 knockdown abolished $\mathrm{Mn}^{2+}$ quench in FDB fibres following store depletion (Fig. 2f), whereas no effect was observed for negative control siRNAs (Fig. 2g). Average ( \pm s.e.m.) values for the maximal rate of $\mathrm{Mn}^{2+}$ quench for the conditions shown in Fig. 1a-g are summarized in Fig. 1h. These results demonstrate that SOCE in adult skeletal muscle requires STIM1-Orail coupling and that this process is abolished by the expression of dnOrail.

Effect of SOCE on $\mathrm{Ca}^{2+}$ transients during tetanic stimulation. As SR Ca ${ }^{2+}$ stores in FDB fibres are significantly reduced during a single high-frequency stimulation train ${ }^{31}$, we determined the effect of inhibiting SOCE on myoplasmic $\mathrm{Ca}^{2}+$ transients elicited by multiple repetitive, high-frequency tetanic stimulation trains (Fig. 3). Myoplasmic $\mathrm{Ca}^{2}+$ transients were monitored in single FDB fibres using mag-fluo-4, a rapid, low-affinity $\mathrm{Ca}^{2}+$ dye that maximizes resolution of $\mathrm{Ca}^{2}+$ transient magnitude and kinetics $^{32}$. Mag-fluo-4-loaded fibres were stimulated for 60 consecutive tetanic trains $(500 \mathrm{~ms}$, every $2.5 \mathrm{~s}$, at $50 \mathrm{~Hz}, 60$ times). $\mathrm{Ca}^{2}+$ transient magnitude during each tetani and the integral of the decaying phase of the $\mathrm{Ca}^{2+}$ transient ('tail $\mathrm{Ca}^{2+}$ transient integral') following termination of each train were quantified (Supplementary Fig. S2a).

In FDB fibres perfused with control Ringer's solution, peak tetanic $\mathrm{Ca}^{2}+$ transient amplitude decreased progressively from the first train, reaching $\sim 50 \%$ by the 60 th train (Fig. $3 \mathrm{a}$ ). In the presence of $0.2 \mathrm{mM} \mathrm{LaCl}_{3}$ plus $0.5 \mathrm{mM} \mathrm{CdCl}_{2}(\mathrm{La} / \mathrm{Cd})$, the amplitude of the first tetanic $\mathrm{Ca}^{2}+$ transient was not significantly different from that observed in control, consistent with the a

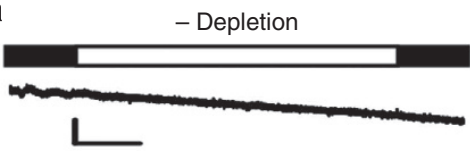

b

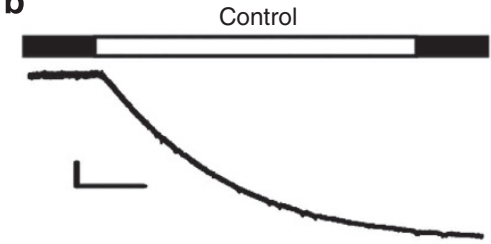

C

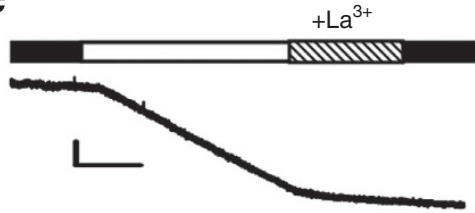

d

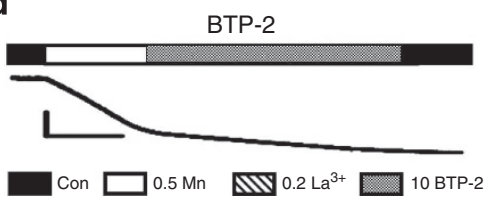

e

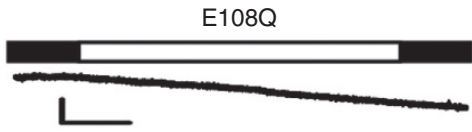

f

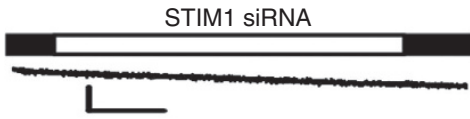

g
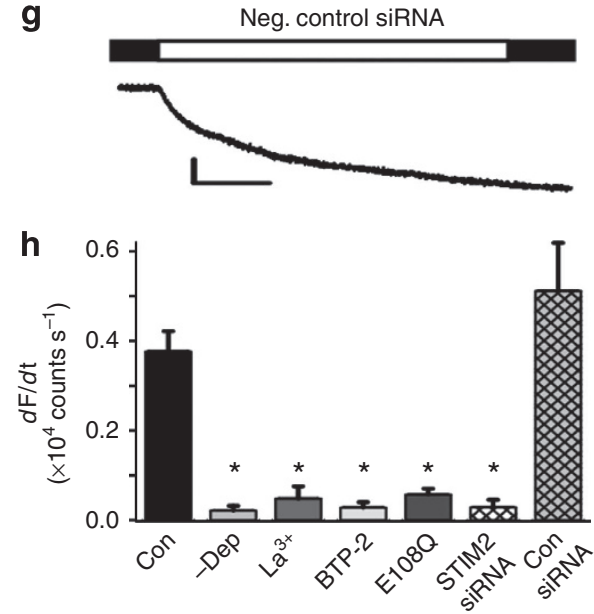

Figure 2 | SOCE in adult skeletal muscle depends on STIM1-Orai1 coupling. (a) Representative $\mathrm{Mn}^{2+}$ quench trace in an adult FDB fibre in the absence of store depletion. (b-g) Representative $\mathrm{Mn}^{2+}$ quench traces in adult FDB fibres obtained following TG-induced store depletion in control (b), during addition of $200 \mu \mathrm{M} \mathrm{La}{ }^{3}$ (c), during addition of $10 \mu \mathrm{M} \mathrm{BTP}-2$ (d), following expression of cherry-tagged E108Q (e), STIM1 siRNAs (f) or negative control siRNAs (g). (h) Average ( \pm s.e.m.) maximum rate of $\mathrm{Mn}^{2+}$ quench in the absence of store depletion ( - Dep, $\left.n=4\right)$ and following TG-induced store depletion in control (Con, $n=29), 200 \mu \mathrm{M} \mathrm{La}^{3+}(n=4), 10 \mu \mathrm{M}$ BTP-2 $(n=10)$, cherry-tagged E108Q expression ( $\left.n=12\right)$, STIM1 siRNAs $(n=8)$ and negative control siRNAs $(n=9) .{ }^{\star} P<0.01$ compared with control by analysis of variance and post hoc Student-Newman-Keuls test. Scale bars, $100 \mathrm{~s}(\mathbf{a}-\mathbf{e}), 50 \mathrm{~s}(\mathbf{f}, \mathbf{g})$ (horizontal); $0.2 \times 10^{6}$ counts $(\mathbf{a}-\mathbf{e}), 0.1 \times 10^{6}$ counts $(\mathbf{f}, \mathbf{g})$ (vertical). 
a
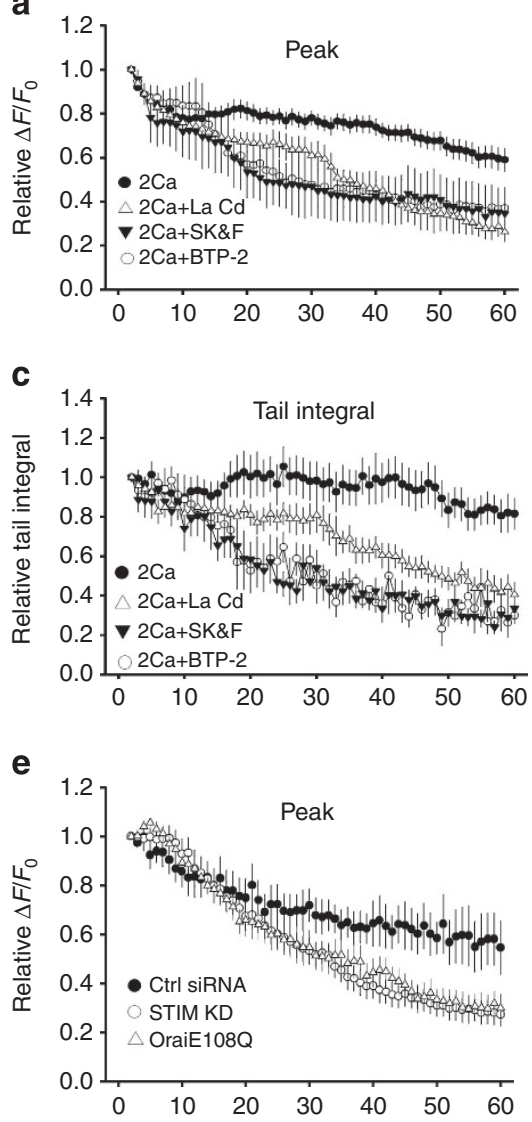

g

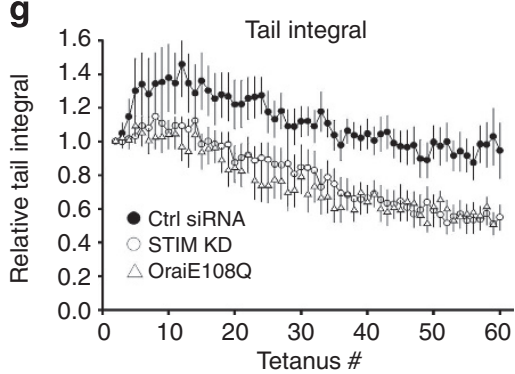

b

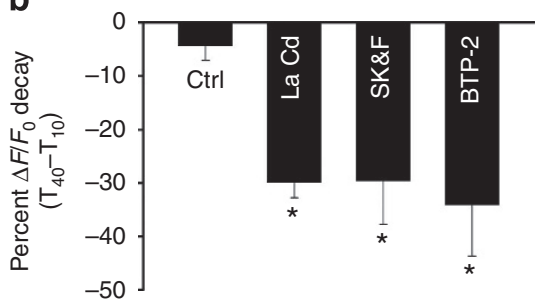

d

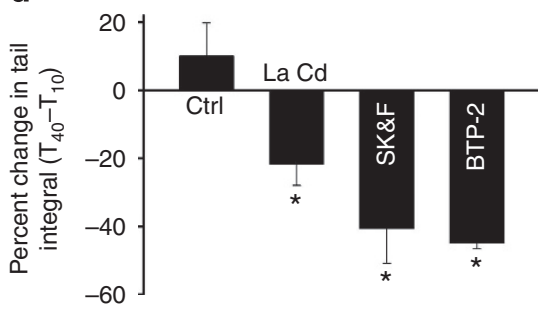

f

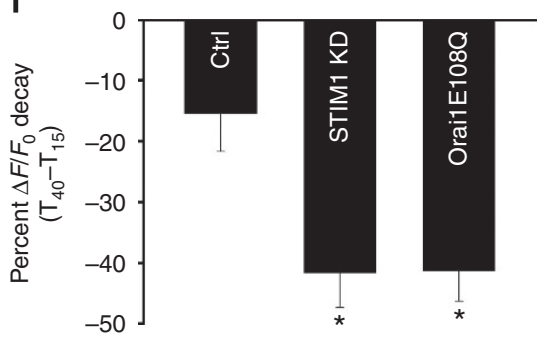

h

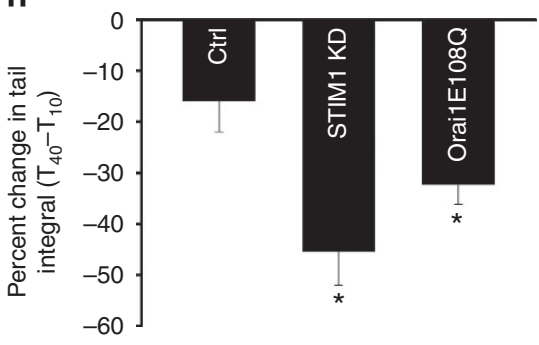

Figure 3 | SOCE maintains $\mathbf{C a}^{2}+$ transient amplitude during repetitive tetanic stimulation. (a) Average ( \pm s.e.m.) peak $\mathrm{Ca}^{2+}$ transient amplitude during 60 consecutive tetanic stimuli $(500 \mathrm{~ms}$, at $50 \mathrm{~Hz}$, every $2.5 \mathrm{~s})$ in control $\left(2 \mathrm{Ca}^{2+}\right.$, filled circles, $\left.n=14\right)$ or in the presence of $200 \mu \mathrm{M} \mathrm{La}{ }^{3+}$ plus

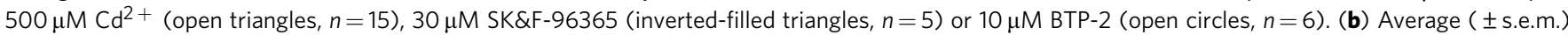
percent reduction in peak $\mathrm{Ca}^{2}+$ transient amplitude from tetanus 10 to tetanus $40\left(\left[\mathrm{~T}_{40}-\mathrm{T}_{10}\right] \times 100\right)$ in the absence and presence of blockers.

(c) Average ( \pm s.e.m.) relative $\mathrm{Ca}^{2+}$ transient tail integral after each tetani for the experiments in shown in a. (d) Average ( \pm s.e.m.) percent change in $\mathrm{Ca}^{2+}$ transient tail integral $\left(\left[\mathrm{T}_{40}-\mathrm{T}_{10}\right] \times 100\right)$ for the experiments shown in c. (e) Average $\left( \pm\right.$ s.e.m.) peak $\mathrm{Ca}^{2}+$ transient amplitude during 60 consecutive tetanic stimuli ( $500 \mathrm{~ms}$ at $50 \mathrm{~Hz}$ every $2.5 \mathrm{~s}$ ) in FDB fibres treated with either negative control siRNA (Ctrl siRNA, filled circles, $n=10)$, STIM1targeted siRNA (STIM KD, open circles, $n=18$ ) or expressing cherry-tagged Orai1E108Q cDNA (open triangles, $n=5$ ). (f) Average $( \pm$ s.e.m.) percent reduction in peak $\mathrm{Ca}^{2+}$ transient amplitude from tetanus 15 to tetanus $40\left(\left[\mathrm{~T}_{40}-\mathrm{T}_{15}\right] \times 100\right)$ in FDB fibres treated with negative control siRNA or STIM1-targeted siRNA or Orai1E108Q cDNA. (g) Average ( \pm s.e.m.) relative $\mathrm{Ca}^{2+}$ transient tail integral for the experiments shown in $\mathbf{e}$. (h) Average $( \pm$ s.e.m. $)$ percent reduction in $\mathrm{Ca}^{2+}$ transient tail integral $\left(\left[\mathrm{T}_{40}-\mathrm{T}_{10}\right] \times 100\right)$ for the experiments shown in $\mathbf{g}$. ${ }^{\star} P<0.05$ compared with control by analysis of variance and post hoc Student-Newman-Keuls test.

independence of skeletal muscle EC coupling on $\mathrm{Ca}^{2}+$ entry. However, the decrement in tetanic $\mathrm{Ca}^{2}+$ transient magnitude was significantly greater by the 30 th train, and even more so by the 60th train, in the presence of $\mathrm{La} / \mathrm{Cd}$ (Fig. 2a and Supplementary Fig. S2b). As a result, a clear sustained phase of $\mathrm{Ca}^{2}+$ transient amplitude was observed between T10 and T40 under control conditions $(-4.3 \pm 2.8 \%$, average \pm s.e.m. $)$ that was reduced approximately sevenfold in the presence of $\mathrm{La} / \mathrm{Cd}$ $\left(-29.9 \pm 2.8 \%\right.$; Fig. 3b). Similarly, the tail $\mathrm{Ca}^{2}+$ transient integral, which reflects a balance between $\mathrm{Ca}^{2+}$ influx and removal and increased $(+10.2 \pm 9.6 \%$, average \pm s.e.m. $)$ between $\mathrm{T} 40$ and $\mathrm{T} 10$ in control (T40 - T10), was reduced in the presence of $\mathrm{La} / \mathrm{Cd}(-21.7 \pm 6.2 \%$; Fig. $3 \mathrm{c}, \mathrm{d})$.

As $\mathrm{La} / \mathrm{Cd}$ blocks multiple forms of $\mathrm{Ca}^{2+}$ entry, effects of more targeted SOCE interventions were also determined. A similar accelerated reduction in peak $\mathrm{Ca}^{2+}$ transient amplitude (Fig. 3a,b) and tail integral (Fig. 3c,d) during repetitive tetanic stimulation was observed following addition of SOCE channel 
inhibitors SK\&F-96365 $(30 \mu \mathrm{M})$ and BTP-2 $(10 \mu \mathrm{M})$. Most importantly, a faster decay in peak $\mathrm{Ca}^{2}+$ transient amplitude (Fig. 3e,f) and tail $\mathrm{Ca}^{2+}$ transient integral (Fig. 3g,h) was also observed in FDB fibres following STIM1 knockdown (but not for control siRNA) and transient expression of Orai1E108Q mutant. The results in Fig. 3 indicate that SOCE has an important role in maintaining $\mathrm{Ca}^{2}+$ transient amplitude during repetitive, high-frequency tetanic stimulation.

Characterization of muscle-specific dnOrail transgenic mice. SOCE is abolished in myotubes ${ }^{12}$ and FDB fibres following transient expression of OrailE108Q (Fig. 2e,h). Therefore, to investigate the role of Orail-dependent $\mathrm{Ca}^{2+}$ entry in adult skeletal muscle, we generated muscle-specific dnOrail (E108Q) transgenic mice (Fig. 4a; see Methods). Several transgenic lines exhibiting either low (line 102), medium (line 5) or high but variable (line 86) dnOrail expression were generated (Fig. 4b). Expression of the dnOrail protein assessed using an antihaemagglutinin (HA) antibody was highly skeletal muscle specific, with no detectable expression in the heart, spleen, liver, lung or kidney (Fig. 4c). The dnOrail protein was partially glycosylated as the doublet band observed on western blot was consolidated to a single band following de-glycosylation with PNGaseF (Fig. 4c). Immunocytochemistry of FDB fibres from line-5 mice using an anti-HA antibody showed a double-rowed a

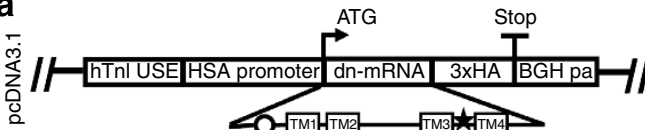

b

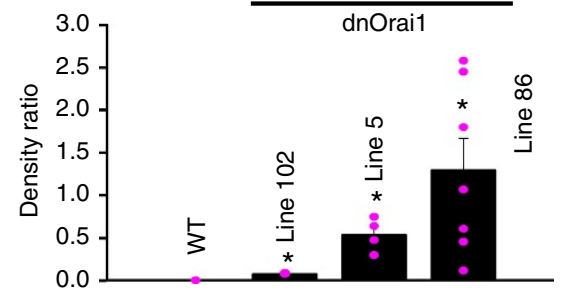

c

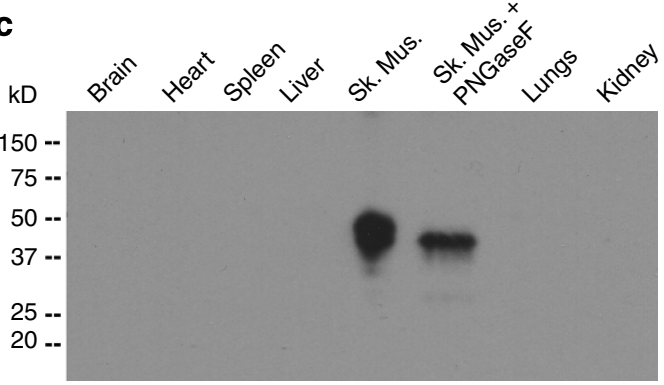

d

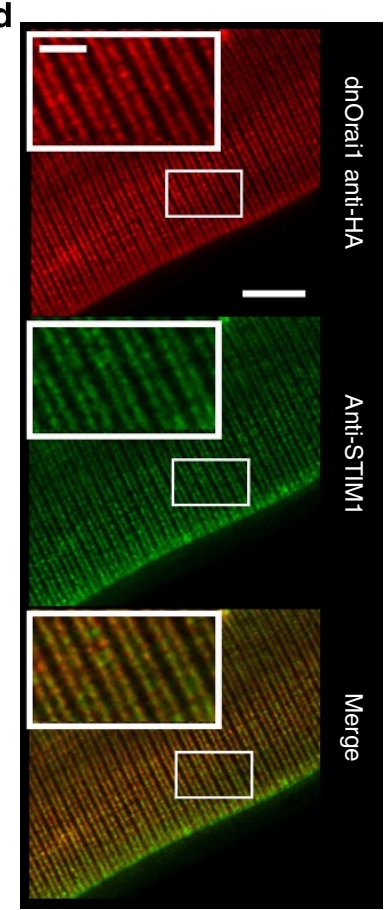

\section{e}

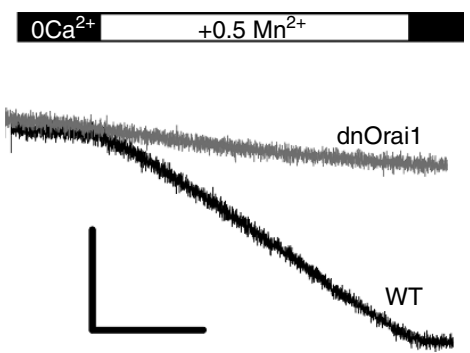

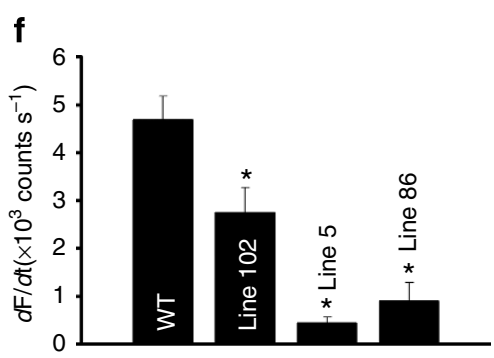

dnOrai1

Figure 4 | Generation of muscle-specific dnOrai1 transgenic mice. (a) Schematic representation of the transgene vector used to create multiple lines of muscle-specific dnOrai1 mice. The dominant-negative E108Q point mutation and a C-terminal $3 \times \mathrm{HA}$ epitope were engineered into a mouse Orai1 cDNA and then cloned into a transgene vector containing a human troponin I upstream enhancer (hTnIUSE), a human skeletal muscle actin (HSA) promoter and a $3^{\prime}$ bovine growth hormone polyadenylation (BGHpa) tail. (b) Relative HA-tagged dnOrai1 protein expression in TA muscles from WT $(n=3)$ and dnOrai1 transgenic lines $102(n=3), 5(n=4)$ and $86(n=7)$ probed by an anti-HA antibody using western blot analysis. HA immunoreactivity was normalized to SERCA. Pink filled circles $(\bullet)$ indicate the relative expression of dnOrai1 in every individual mouse analysed. ${ }^{\star} P<0.05$ by analysis of variance followed by Dunn's post hoc test. Line-5 mice exhibit uniform moderate transgene expression, whereas line- 86 mice exhibit high but more variable tansgene expression. (c) Western blot analysis with an anti-HA antibody showing skeletal muscle-specific expression of dnOrai1 protein in line-5 mice. (d) Imunonocytochemical labelling of dnOrai1 with anti-HA antibody (top), endogenous STIM1 (middle) and a merged image showing co-localization of the two proteins in double rows of striated fluorescence reflecting triad distribution (bottom). Scale bar, $10 \mu \mathrm{m}$. (e) Representative $\mathrm{Mn}^{2+}$ quench traces in TG-treated FDB fibres obtained from WT and line-86 dnOrai1 mice. Scale bars, $100 \mathrm{~s}$ (horizontal), $4 \times 10^{5}$ counts (vertical). (f) Average ( \pm s.e.m.) maximum rate of $\mathrm{Mn}^{2+}$ quench in TG-treated FDB fibres from WT $(n=9)$, line-102 $(n=13)$, line-5 $(n=15)$ and line-86 $(n=7)$ dnOrai1 mice.

${ }^{\star} P<0.05$ compared with WT by Student's $t$-test. 
striated pattern that co-localized with STIM1 fluorescence (Fig. 4d), similar to that observed for endogenous Orail (Fig. 1a). Importantly, FDB fibres obtained from both line-86 (Fig. 4e,f) and line-5 (Fig. 4f) mice lacked SOCE as assessed by $\mathrm{Mn}^{2+}$ quench.

Unlike that observed following global or muscle-specific STIM1 ablation ${ }^{11,13}$, line-5 muscle-specific dnOrail transgenic mice were born at normal Mendelian ratios, thrived into adulthood and exhibited normal activity and fertility during the course of study up to 10 months of age. Similar to STIM1 knockout mice, line-5 dnOrail mice exhibited a significant reduction in whole-body mass at birth compared with wild-type (WT) littermates, which persisted throughout postnatal development and into adulthood (Fig. 5a). Muscle mass of tibialis anterior (TA), extensor digitorum longus (EDL) and soleus muscles were all significantly reduced ( $\sim 20-30 \%)$ in line-5 dnOrail mice compared with WT littermates (Fig. 5b). Analysis of muscle cross-sectional area (CSA) by dystrophin immunolabelling revealed a significant reduction $(\sim 33 \%)$ in average fibre CSA in TA muscles of line-5 dnOrail mice (Fig. 5c,d). Nevertheless, haematoxylin and eosin (H\&E) staining of TA muscle sections showed no evidence of muscle damage, degeneration, central nucleation or infiltration of inflammatory fibres from line-5 dnOrail mice (Fig. 5c, bottom). In addition, myosin heavy chain analyses revealed no change in the proportion of type I, IIa, IIx or IIb fibres in TA, EDL, soleus and FDB muscles (Fig. 5e,f). Using electron microscopy, the ultrastructure, myofibril spacing and disposition of SR, transverse a

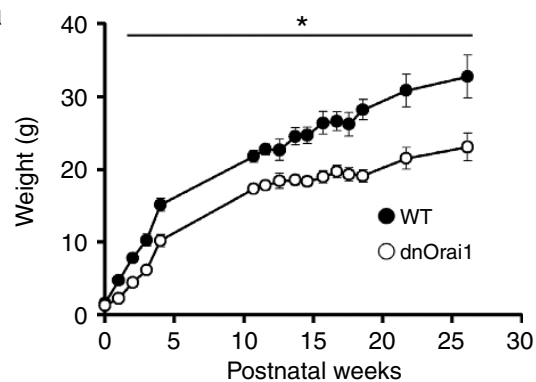

C

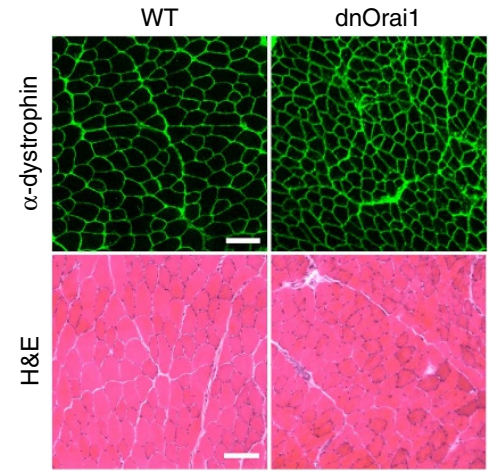

e
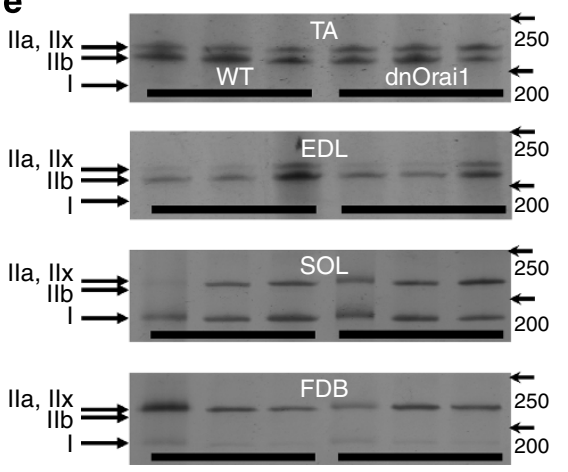

b

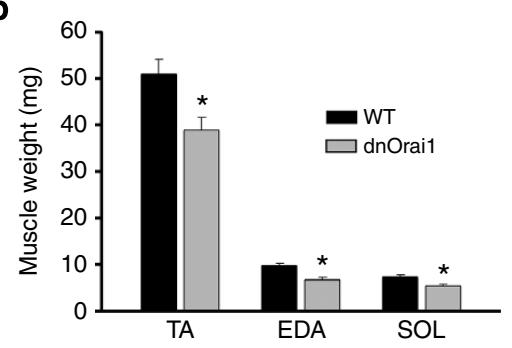

d

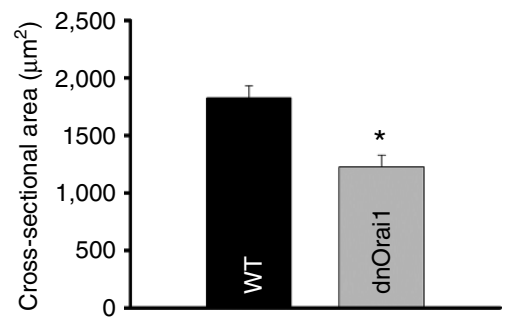

$\mathbf{f}$

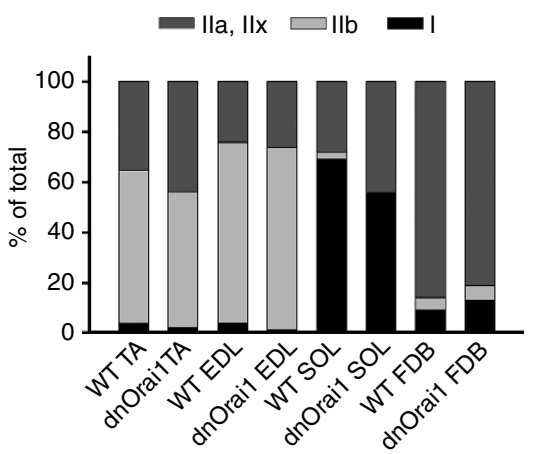

Figure 5 | Reduced weight and fibre CSA in dnOrai1 transgenic mice. (a) Postnatal growth curves (birth to 6 months) obtained from line- 5 mice showing a significant reduction in total body weight of muscle-specific dnOrai1 transgenic mice compared with WT littermate controls ( $n=5$ female mice with both WT and dnOrai1). ${ }^{\star} P<0.01$ compared with WT by Student's $t$-test. (b) Average ( \pm s.e.m.) muscle weight of TA, EDL and soleus in line- 5 dnOrai1 transgenic mice ( $n=5$ mice) compared with that of WT littermate controls ( $n=7$ mice). ${ }^{\star} P<0.05$ compared with corresponding WT control by Student's $t$-test. (c) Representative dystrophin immunostaining (top panel) and H\&E images (bottom panel) of TA muscle cross-sections from WT and line-5 dnOrai1 mice. Scale bars, $100 \mu \mathrm{m}$. (d) Average ( \pm s.e.m.) fibre CSA calculated from dystrophin-stained TA muscle (as shown in c) from WT $(n=4)$ and line- 5 dnOrai1 mice $(n=3) .{ }^{\star} P<0.05$ by Student's $t$-test. (e) Representative silver-stained polyacrylamide gel images of myosin heavy chain analysis from TA, EDL soleus and FDB of three different WT and line- 5 dnOrai1 mice. Molecular weight markers are indicated to the right of each gel. (f) Stacked bar graph showing average fast Ila, Ilx, Ilb and slow type I fibre composition in WT and line-5 dnOrai1 transgenic mice ( $n=3-5$ for both WT and dnOrai1). No significant difference in fibre-type composition was observed between WT and dnOrai1 mice. 

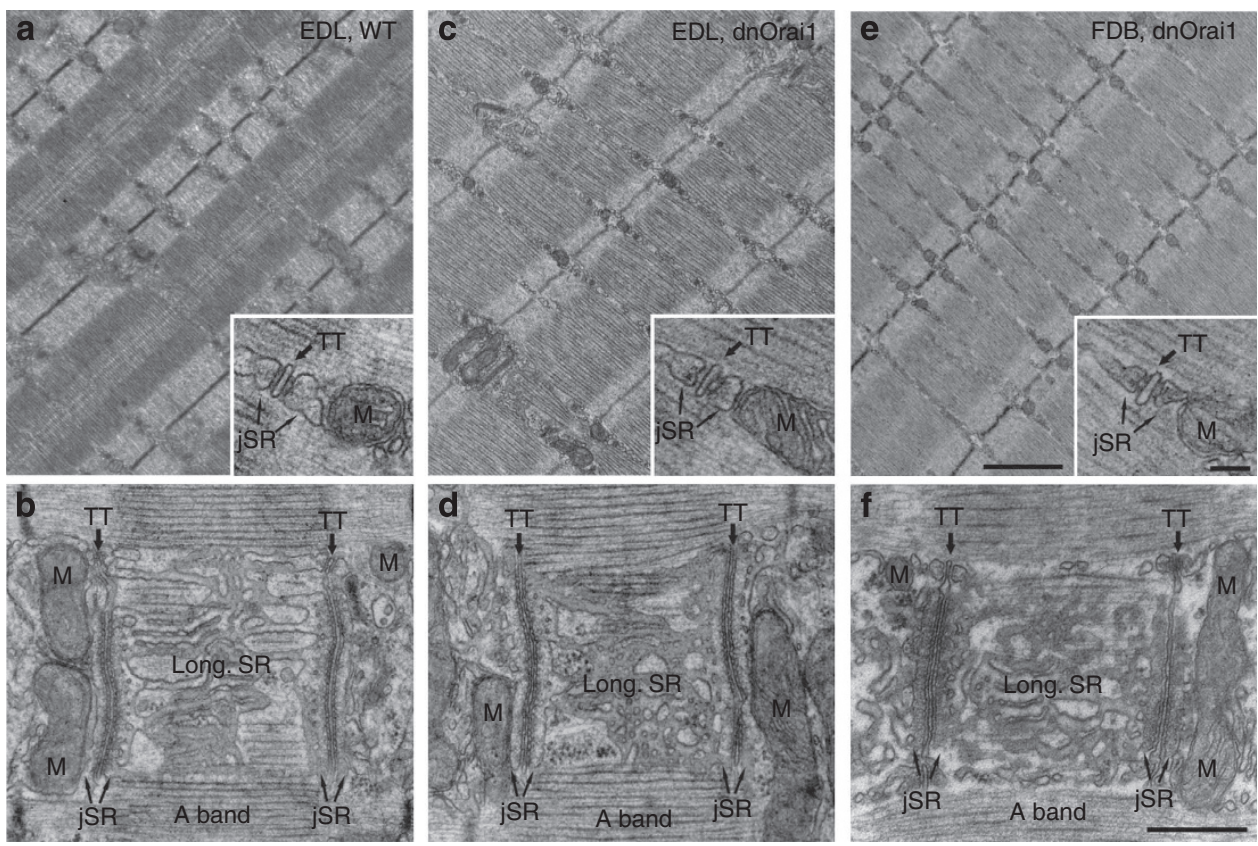

Figure 6 | Preserved skeletal muscle ultrastructure in EDL and FDB muscle of line-5 dnOrai1 mice. Representative electron microscopy micrographs from WT EDL $(\mathbf{a}, \mathbf{b})$, line-5 dnOrai1 EDL (c,d) and line-5 dnOrai1 FDB (e,f), with all samples exhibiting similar ultrastructure. Sarcomeres of adjacent myofibrils are similarly well aligned (a,c,e), with triads and the mitochondria closely associated (insets). Junctional SR (jSR) is closely associated with transverse tubules (TT) adjacent to the sarcomere I-A band transition, whereas longitudinal SR (long. SR) is placed at the A band (images are close to the surface of a myofibril; $\mathbf{b}, \mathbf{d}, \mathbf{f}$ ). Mitochondria (M) are in close proximity of jSR/TT junctions (triads) on the side opposite to long. SR in fibres from both WT (b) and dnOrai1 (d,f) mice. Scale bars, $1 \mu \mathrm{m}(\mathbf{a}, \mathbf{c}, \mathbf{e}) ; 0.5 \mu \mathrm{m}(\mathbf{b}, \mathbf{d}, \mathbf{f}) ; 0.1 \mu \mathrm{m}$ (insets).

tubules, triads and the mitochondria in EDL and FDB fibres from line-5 dnOrail mice were indistinguishable from WT (see Fig. 6 for additional details). Only a few fibres from line-5 dnOrail mice (3/63) exhibited minor structural defects (limited regions of Z-line streaming or a few swollen mitochondria).

Western blot analysis using an Orail-specific antibody showed no change in endogenous Orail expression in TA muscles of dnOrail mice (Supplementary Fig. S3a). Expression of STIM1 (Supplementary Fig. S3b) and other proteins involved in $\mathrm{Ca}^{2+}$ homoeostasis (that is, sarco/endoplasmic $\mathrm{Ca}^{2+}$ ATPase 1, RyR1, calsequestrin type 1 and dihydropyridine receptor $\alpha 1$-subunit; Supplementary Fig. S4) were also unaltered in TA muscles of dnOrail mice. Consistent with this, the magnitude and decay kinetics of electrically evoked twitch $\mathrm{Ca}^{2+}$ release was not different in single FDB fibres from adult WT and dnOrail mice (Supplementary Fig. S5a-c). However, total $\mathrm{Ca}^{2+}$ store content assessed using a rapid $\mathrm{Ca}^{2+}$ store release cocktail ${ }^{33}$ was significantly reduced $(P<0.01$, Student's $t$-test $)$ in FDB fibres from dnOrail mice (fura-2FF $\Delta$ ratio was $0.36 \pm 0.03$, average \pm s.e.m. $n=11$ and $0.17 \pm 0.05, n=6$ for WT and dnOrail, respectively; Supplementary Fig. S5d,e).

dnOrail mice exhibit increased susceptibility to fatigue. As muscle-specific dnOrail mice thrive into adulthood and lack SOCE in skeletal muscle (Fig. 4e,f), these mice provide a unique opportunity to determine the physiological role of Orail-dependent SOCE in adult muscle. As an initial test of the functional impact of loss of SOCE in muscle, we compared ex vivo muscle contractility of EDL muscles isolated from 4-6-month old WT and line- 5 dnOrail mice. The magnitude and kinetics of EDL twitch contraction were not different between WT and dnOrail (Fig. $7 \mathrm{a}$, left traces), consistent with the normal fibre-type distribution, ultrastructure and EC coupling in muscles from
dnOrail mice. However, a modest reduction in peak musclespecific force was observed in EDL muscles from dnOrail mice at stimulation frequencies $\geq 100 \mathrm{~Hz}$ (Fig. 7a,b). EDL muscles from dnOrail mice also exhibited a reduced ability to maintain contractile force at high frequencies during each $500 \mathrm{~ms}$ stimulation (Fig. 7a, grey traces and Fig. 7c), consistent with an increase in EDL fatigability during intense stimulation. Moreover, the magnitude of force decay during a prolonged tetanus $(150 \mathrm{~Hz}, 2 \mathrm{~s})$ was increased in EDL muscles from dnOrail mice (Fig. 7d,e).

We next compared the ability of muscles from dnOrail mice to produce sustained $\mathrm{Ca}^{2+}$ release and force production during repetitive high-frequency tetanic stimulation (Fig. 8a-d). Single FDB fibres from WT and dnOrail mice were subjected to the same repetitive tetanic stimulation train used for the experiments shown in Fig. $3(50 \mathrm{~Hz}, 500 \mathrm{~ms}$, every $2.5 \mathrm{~s}, 60$ times). Consistent with results obtained with SOCE channel inhibitors, STIM1 $\mathrm{KD}$, and OrailE108Q expression, peak tetanic $\mathrm{Ca}^{2+}$ transient decline was accelerated in FDB fibres from dnOrail mice (Fig. 8a). Peak Ca ${ }^{2+}$ transient amplitude decreased $47.0 \pm 7.3 \%$ (average \pm s.e.m.) from $\mathrm{T} 5$ to $\mathrm{T} 40$ in fibres from dnOrail mice, in contrast to only $15.6 \pm 9.6 \%$ for fibres from WT mice (Fig. 8b). A similar accelerated decline in force production repetitive tetanic stimulation was also observed in EDL muscles from dnOrail mice (Fig. 8c) where tetanic force during the first five tetani decreased $14.6 \pm 2.7 \%$ (average \pm s.e.m.) for dnOrail compared with only $7.4 \pm 1.6 \%$ for WT (Fig. 8d). A similar decrease in peak-specific force and increased fatigability during repetitive stimulation were observed in EDL muscles from dnOrail line-86 mice (Supplementary Fig. S6) Altogether, these results are consistent with a significant contribution of SOCE to sustained $\mathrm{Ca}^{2+}$ release and force production during repetitive, high-frequency stimulation.

Finally, dnOrail mice were used to assess the consequences of loss of SOCE function on muscle performance at the 

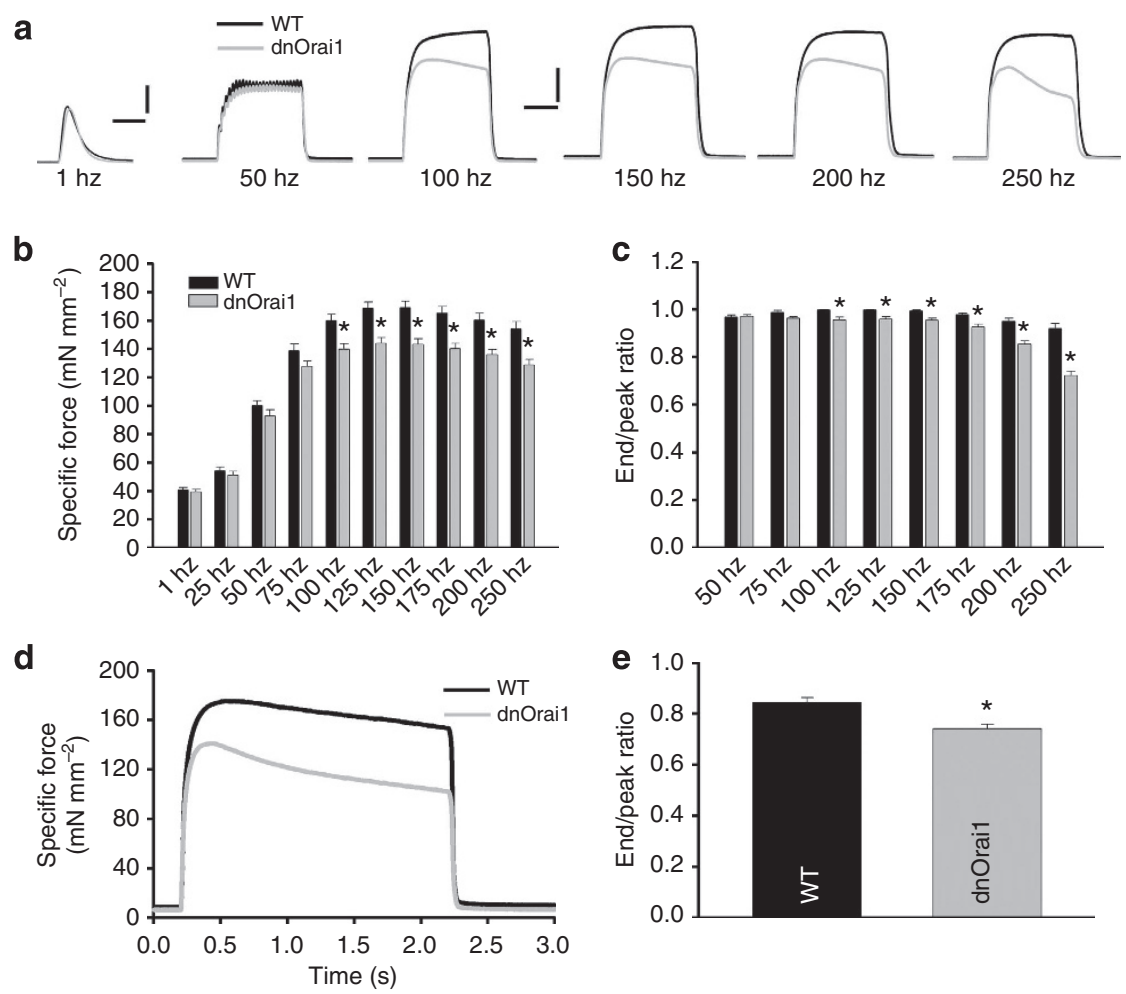

Figure 7 | Altered maximum tetanic-specific force and contractile decay in EDL muscles of dnOrai1 mice. (a) Representative superimposed contraction traces elicited at different stimulation frequencies (1,50, 100, 150, 200 and $250 \mathrm{~Hz}$ ) in EDL muscles from WT (black) and line-5 dnOrai1 (grey) mice. (b) Frequency dependence of average ( \pm s.e.m.) specific force $\left(\mathrm{mN} \mathrm{mm}^{-2}\right)$ in EDL muscles from WT (black, $\left.n=14\right)$ and line-5 dnOrai1 mice (grey, $n=14)$. ${ }^{\star} P<0.01$ compared with corresponding control by Student's $t$-test. (c) Frequency dependence of average ( \pm s.e.m.) contractile decay (end/ peak ratio) for EDL muscles from WT (black bars, $n=14$ ) and line- 5 dnOrai1 (grey bars, $n=14$ ). ${ }^{\star} P<0.01$ compared with corresponding control by Student's t-test. (d,e) Representative contractile traces (d) and average contractile decay (end/peak ratio; e) during a single $2 \mathrm{~s}$, $150 \mathrm{~Hz}$ stimulation train in EDL muscles from WT (black, $n=10$ ) and line- 5 dnOrai1 mice (grey, $n=11$ ). ${ }^{\star} P<0.01$ compared with WT by Student's $t$-test. Scale bars, 50 ms (left, for twitch only, horizontal); $20 \mathrm{mN} \mathrm{mm}^{-2}$ (vertical); $200 \mathrm{~ms}$ (right, for tetani, horizontal); $50 \mathrm{mN} \mathrm{mm}^{-2}$ (vertical).

whole-animal level. Age- (4-5-month-old male) and sex-matched WT and line-5 dnOrail mice were evaluated using a prolonged Rotarod protocol designed to assess endurance. In this test, mice were placed on a motor driven Rotarod wheel and forced to walk on the rotating wheel for $2 \mathrm{~h}$ while the speed was increased modestly at regular intervals (see Methods). WT mice remained on the wheel with only a few falls during the first hour, with progressively more falls during the second hour. On the other hand, although initially performing equally well at the task, dnOrail mice exhibited a significant increase in the number of cumulative falls compared with WT mice at all times $30 \mathrm{~min}$ after initiation of the task (Fig. 8e and Supplementary Movie 1). Because of exhaustion, several dnOrail mice were unable to complete the entire $2 \mathrm{~h}$ protocol. Thus, only the cumulative number of falls during the first $60 \mathrm{~min}$ of the task is shown in Fig. 8e. As a second measure of fatigue, a separate group of WT and line- 5 dnOrail mice were subjected to three consecutive days of acute treadmill running (see Methods). All WT and dnOrail mice completed the entire $1 \mathrm{~km}$ run on each of three consecutive days. However, WT mice completed the task with limited rests and encouragement. On the other hand, after approximately the first $40 \mathrm{~min}$ of running, dnOrail mice exhibited a significant increase in the number of rests and required more encouragement to re-engage the treadmill and continue running (Fig. $8 \mathrm{f}$ and Supplementary Movie 2). Altogether, results from the Rotarod exhaustion and treadmill-running tasks indicate that adult dnOrail mice exhibit a significantly increased susceptibility to muscle fatigue.

\section{Discussion}

This study characterized the structural basis and physiological role of SOCE in adult skeletal muscle. Immunocytochemical results provide direct evidence for co-localization of endogenous Orail and STIM1 in the triad junction and $\mathrm{Mn}^{2+}$ quench studies found that STIM1-Orail coupling is required for SOCE in adult skeletal muscle. This close disposition of STIM1 and Orai1 in existing SR-T-tubule triad junctions enables rapid SOCE activation as STIM1 and SR redistribution following store depletion is not required. However, results of BiFC experiments indicate that preformed STIM1-Orail complexes are not present in resting muscle fibres with fully replete $\mathrm{SR} \mathrm{Ca}^{2+}$ stores and that store depletion is required to drive the formation of junctional OrailSTIM1 complexes. FRET-based studies will be required to further resolve the time course of junctional STIM1-Orail complex formation during SR $\mathrm{Ca}^{2+}$ store depletion.

The survival of muscle-specific dnOrail mice allowed us to assess the physiological role of Orail-dependent SOCE in adult skeletal muscle. Although line- 5 dnOrail mice lack SOCE, these mice thrive into adulthood and exhibit normal muscle histology and fibre-type distribution. Thus, Orail-dependent SOCE is clearly not required for survival. However, as muscle mass and CSA are reduced in line- 5 dnOrail mice, SOCE is required for normal muscle growth. Although high expressing line-86 mice are fertile, live well into adulthood and exhibit a similar reduction in muscle mass and CSA, some of these mice exhibit histopathological signs of myopathy including increased cell infiltration, central nucleation and the development of kyphosis at 

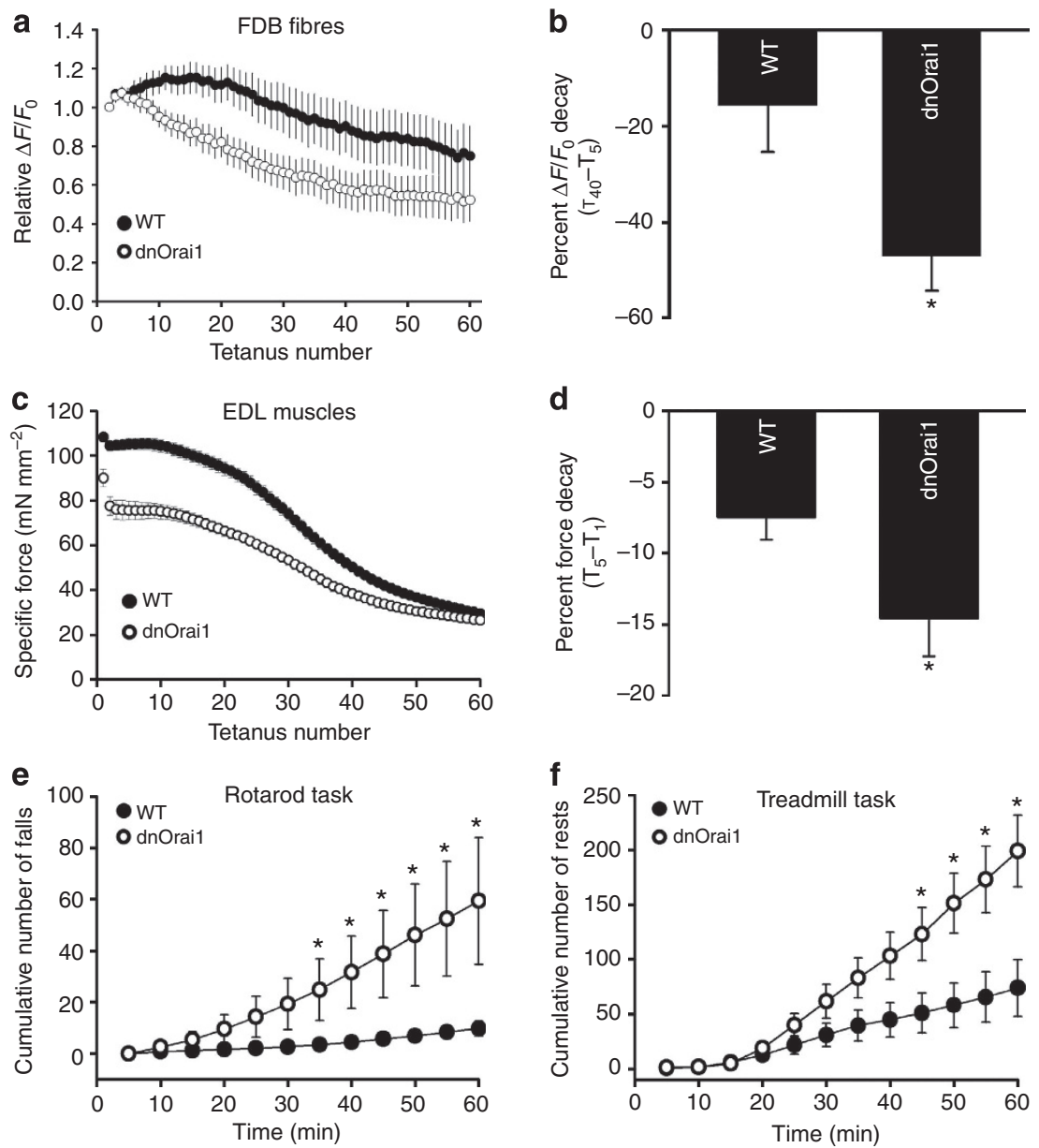

Figure 8 | dnOrai mice exhibit increased susceptibility to fatigue. (a) Average ( \pm s.e.m.) peak $\mathrm{Ca}^{2+}$ transient amplitude during 60 consecutive tetanic stimuli $(500 \mathrm{~ms}$ at $50 \mathrm{~Hz}$ every $2.5 \mathrm{~s}$ ) in FDB fibres obtained from either WT (filled circles, $n=11$ ) or line-86 dnOrai1 transgenic mice (open circles, $n=15)$. (b) Average ( \pm s.e.m.) percent reduction in peak Ca ${ }^{2+}$ transient amplitude from tetanus 5 to tetanus $40\left(\left[T_{40}-T_{5}\right] \times 100\right)$ for the experiments shown in a. ${ }^{\star} P<0.05$ by Student's $t$-test. (c) Average ( \pm s.e.m.) peak-specific force during 60 consecutive tetanic stimuli $(500 \mathrm{~ms}$, at $50 \mathrm{~Hz}$, every $2.5 \mathrm{~s})$ in EDL muscles from WT (filled circles, $n=8$ muscles from four mice) or line- 5 dnOrai1 mice (open circles, $n=8$ muscles from five mice). (d) Average ( \pm s.e.m.) percent force decay during the first five successive tetani $\left(100 \times\left[T_{5}-T_{1}\right] / T_{1}\right)$ for the experiments shown in $\mathbf{c}$. ${ }^{\star} P<0.05$ by Student's $t$-test. (e) Average ( \pm s.e.m.) cumulative number of falls during $1 \mathrm{~h}$ Rotarod endurance task in 4-month-old adult WT $(n=11)$ and line-5 dnOrail $(n=9)$ mice (see Methods). ${ }^{\star} P<0.05$ by Student's $t$-test. (f) Average ( \pm s.e.m.) cumulative number of rests (that is, all four paws off the treadmill) observed during $1 \mathrm{~km}$ treadmill run in 4-month-old adult WT $(n=6)$ and line- 5 dnOrai1 $(n=5)$ mice (see Methods). ${ }^{\star} P<0.05$ by Student's $t$-test. The phenotypes (absence of SOCE, reduced body and muscle weight, increased fatigue, reduced treadmill running) of line-5 mice on the mixed background (B6SJL hybrid) was the same as that observed for line- 5 mice following backcross onto a congenic C57BI6 background.

advanced ages. The myopathic phenotypes observed in line-86 mice is unlikely due to loss of Orail-dependent SOCE, as they are not observed in line- 5 mice that also lack SOCE. The reasons for the variable myopathic alterations observed in line- 86 dnOrail mice is unclear but may involve off-target effects (for example, inhibition of TrpC channels) ${ }^{34}$ of the high level of dnOrail expression observed in some of these mice (Fig. 4b).

The role of SOCE in refilling SR $\mathrm{Ca}^{2+}$ stores in skeletal muscle is controversial. On the one hand, SR $\mathrm{Ca}^{2+}$ content was reported to be increased in FDB fibres from $m d x$ mice $^{35}$ where Orai $1^{35,36}$ and STIM1 (ref. 36) protein levels are upregulated and SOCE is enhanced, whereas SR $\mathrm{Ca}^{2+}$ store content is reduced in muscle-specific STIM1 knockout mice that lack SOCE ${ }^{11}$. However, as other critical $\mathrm{Ca}^{2+}$-handling proteins (for example, SERCA, TrpC3/6 and NCX3) are also altered in these models ${ }^{11,35}$, the specific role of STIM1-Orail coupling in store refilling remains unclear. In addition, Launikonis et al. ${ }^{25}$ reported that in spite of changes in STIM1 and Orail expression in $m d x$ muscle, SR $\mathrm{Ca}^{2+}$ content was unaltered in mechanically skinned
EDL muscle fibres ${ }^{36}$, and thus concluded that SOCE has little or no role in refilling SR $\mathrm{Ca}^{2+}$ stores in skeletal muscle $25,36,37$. However, as SR reloading in mechanically skinned fibres may be different from that of intact fibres, the relevance of these results to $\mathrm{Ca}^{2+}$ store content in intact fibres under more physiological conditions is unclear. Our finding that resting $\mathrm{Ca}^{2}+$ store content was significantly reduced in intact single FDB fibres from dnOrail mice is consistent with Orail-dependent $\mathrm{Ca}^{2+}$ entry having a role in maintaining and refilling $\mathrm{SR} \mathrm{Ca}^{2}+$ stores as it does in non-excitable cells. In addition, as no alterations in other major $\mathrm{Ca}^{2+}$-handling proteins were found in the muscle from dnOrail mice (Supplementary Fig. S4), our findings provide the strongest evidence to date that Orail-dependent SOCE contributes to store refilling in the skeletal muscle.

The reduction in total $\mathrm{Ca}^{2+}$ store content likely contributes to the reduced peak tetanic-specific force and accelerated fatigue with repetitive high-frequency stimulation. During repetitive tetanic stimulation of FDB fibres from WT mice, peak $\mathrm{Ca}^{2+}$ transient magnitude is maintained and $\mathrm{Ca}^{2+}$ transient tail 
integral is increased, both of which are reduced by SOCE inhibitors, STIM1 KD, and dnOrail expression. These findings indicate that repetitive tetanic stimulation promotes store depletion and activation of STIM1/Orail-dependent SOCE needed to replenish $\mathrm{SR} \mathrm{Ca}^{2+}$ stores and maintain $\mathrm{Ca}^{2+}$ release during subsequent tetani. Thus, SOCE has an important role in sustaining $\mathrm{Ca}^{2}+$ release and contractile force during repetitive tetanic stimulation, consistent with that reported previously when SOCE activity is reduced either pharmacologically ${ }^{19}$ or in aging ${ }^{38}$. Our findings that adult dnOrail mice exhibit a marked increased susceptibility to exhaustion during prolonged Rotarod and treadmill exercise provide the strongest evidence to date that Orail-dependent SOCE has a key role in limiting muscle fatigue during strenuous exercise.

Muscle-specific dnOrail transgenic mice also provide insight into the role of Orail-dependent SOCE in skeletal muscle development, specification, growth and survival. As reported for muscle-specific STIM1 knockout mice ${ }^{11}$, dnOrail mice exhibit similar reductions in body weight, muscle mass and fibre CSA. Taken together, these findings demonstrate that STIM1-Orail signalling promotes muscle growth in both young and adult mice, presumably through the activation of $\mathrm{Ca}^{2}+$-dependent downstream signalling cascades ${ }^{11}$. However, STIM1 knockout mice also exhibit hypotonia, increased central nucleation, swollen mitochondria and neonatal lethality ${ }^{11,13}$, none of which are observed in line-5 dnOrail mice. These additional phenotypes observed in STIM1 knockout mice, but not in line-5 dnOrail mice, probably reflect altered activity of one or more non-Orail STIM1 targets including $\mathrm{TrpC} / \mathrm{ARC} / \mathrm{Ca}_{\mathrm{V}}$ channels, SERCA/PMCA/NCX pumps/exchangers, adaptor proteins, ER chaperones, second messenger enzymes or ER stress/remodelling proteins $^{23}$.

In summary, we provide a comprehensive characterization of Orail-dependent SOCE in adult skeletal muscle. SOCE in adult muscle depends on junctional STIM1-Orail coupling, which is important for both normal muscle growth and susceptibility to fatigue. Altered SOCE activity has been proposed to contribute to muscle dysfunction in various pathophysiological states of muscle including sarcopenia ${ }^{38}$, muscular dystrophy ${ }^{36}$ and malignant hyperthermia $^{39}$. Muscle-specific dnOrail mice will provide a powerful model to assess the relative importance of Oraildependent SOCE in normal muscle physiology and how SOCE dysfunction contributes to these and other muscle pathologies.

\begin{abstract}
Methods
In vivo electroporation of FDB muscles. All experiments were approved by the University of Rochester Committee on Animal Recourses. Four to six-month-old male and female WT C57Bl6 mice were anaesthetized by intraperitoneal injection of $100 \mathrm{mg} \mathrm{kg}^{-1}$ ketamine, $10 \mathrm{mg} \mathrm{kg}^{-1}$ xylazine and $3 \mathrm{mg} \mathrm{kg}^{-1}$ acepromazine. Hindlimb footpads of anaesthetized mice were then injected with bovine hyalur-

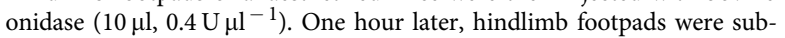
cutaneously injected with either cherry-tagged Orail cDNA construct $(20 \mu \mathrm{g}$ in $71 \mathrm{mM} \mathrm{NaCl}$ ), STIM1-specific siRNAs (Dharmacon siGENOME SMARTpool, $20 \mathrm{pmol}$ in $71 \mathrm{mM} \mathrm{NaCl}$ ) or negative control siRNA (Dharmacon, 20 pmol in $71 \mathrm{mM} \mathrm{NaCl}$, see Supplementary Table S1 for sequence) using a 30-gauge needle. The footpad was then electroporated with 10 stimulations of $100 \mathrm{~V} \mathrm{~cm}^{-1}, 20-\mathrm{ms}$ duration delivered at $1 \mathrm{~Hz}$ using subcutaneous gold-plated electrodes placed perpendicular to the long axis of the muscle, close to the proximal and distal tendons ${ }^{40}$. FDB muscles and fibres were used for experiments either 5-8 days after cDNA injection or boosted with a second round of siRNA electroporation on day 5-7 and then used for experiments 10-12 days after the initial electroporation.
\end{abstract}

Isolation of FDB muscle fibres. FDB muscle fibres were dissociated from FDB muscles from 4-6-month-old male and female mice by enzymatic digestion in $0.1 \%$ collagenase $\mathrm{A}, 45 \mathrm{~min}$ at $37^{\circ} \mathrm{C}$ in Ringer's solution ${ }^{40}$.

Bimolecular fluorescence complementation. BiFC is based on the association of two non-fluorescent fragments of YFP that fuse together to form a functional fluorophore when the fragments were located in close proximity (within $10 \mathrm{~nm}$ ) to each other ${ }^{28-30}$. Thus, a putative interaction between two proteins can be examined by attaching complementary YFP fragments to the two proteins with YFP fluorescence used as a read-out of the interaction. For these experiments, YFP was split into two fragments at amino acid 158. The amino-terminal fragment of YFP (1-158) was fused to the carboxy terminus of cherry-tagged STIM1 and the C-terminal YFP fragment (159-238) was fused to the C terminus of cherry-tagged Orail (Supplementary Fig. 2A). Cherry-STIM1-YFP1-158 and cherry-OrailYFP159-238 cDNAs were electroporated into mouse footpads at a 1:1 ratio. YFP fluorescence was monitored in cherry-expressing FDB fibres before and after store depletion. YFP and cherry were excited sequentially by 488 or $543 \mathrm{~nm}$ lasers, respectively, and fluorescence emission detected at $515 / 30$ or $605 / 75 \mathrm{~nm}$, respectively, with a Nikon Eclipse $\mathrm{C} 1$ Plus Confocal microscope (Nikon Instruments, Melville, NY, USA) using either SuperFluor $\times 40$ (1.3 numerical aperture (NA)) or $\times 60(1.4 \mathrm{NA})$ oil-immersion objectives. Images were analysed using AutoQuant software.

$\mathbf{M n}^{\mathbf{2}}+$ quench measurements. Acutely isolated single FDB fibres were loaded with $5 \mu \mathrm{M}$ fura- $2 \mathrm{AM}$ for $1 \mathrm{~h}$ at $37^{\circ} \mathrm{C}$ in a $\mathrm{Ca}^{2+}$-free rodent Ringer's solution containing (in mM): $145 \mathrm{NaCl}, 5 \mathrm{KCl}, 1 \mathrm{MgCl}_{2}, 10$ HEPES, 0.2 EGTA, pH 7.4. During fura-2 loading, unless otherwise stated, all fibres were simultaneously treated with a SERCA pump inhibitor cocktail consisting of $1 \mu \mathrm{M}$ TG, $15 \mu \mathrm{M}$ cyclopiazonic acid (CPA) and $30 \mu \mathrm{M} N$-benzyl-p-toluene sulphonamide, a skeletal muscle myosin inhibitor used to inhibit movement during electrical stimulation, to deplete SR Ca ${ }^{2+}$ stores, prevent $\mathrm{Ca}^{2+}$ reuptake and activate SOCE. Store-depleted fibres were then plated on glass-bottom dishes, bathed in $\mathrm{Ca}^{2+}$-free Ringer's and excited at $362 \mathrm{~nm}$ (isosbestic point of fura-2), while emission was detected at $510 \mathrm{~nm}$ using a DeltaRam illumination system (Photon Technology, Birmingham, $\mathrm{NJ})$. After obtaining an initial rate of fura-2 quench from $\left(R_{\text {baseline }}\right)$, FDB fibres were then exposed to $0.5 \mathrm{mM} \mathrm{MnCl}_{2}$ to access the rate of $\mathrm{Mn}^{2}+$ entry. The maximum rate of fura-2 quench in the presence of $\mathrm{Mn}^{2+}\left(R_{\max }\right)$ was then obtained from the peak differential of the fura- 2 emission trace during $\mathrm{Mn}^{2}+$ application. The rate of SOCE was calculated as $R_{\mathrm{SOCE}}=R_{\max }-R_{\text {baseline }}$ and expressed as $d \mathrm{~F} / d \mathrm{t}$ in counts $\mathrm{s}^{-1}$ (ref. 12).

High-frequency tetanic stimulation of FDB fibres. Myoplasmic $\mathrm{Ca}^{2+}$ transient in FDB fibres during high-frequency tetanic stimulation were monitored using mag-fluo-4 (ref. 41) throughout the entire repetitive tetanic stimulation train (60 successive $500 \mathrm{~ms}, 50 \mathrm{~Hz}$ tetani every $2.5 \mathrm{~s}$; Supplementary Fig. S2a, top). FDB fibres were perfused with Ringer's solution in the presence or absence of $\mathrm{Ca}^{2+}$ influx blockers. Peak $\mathrm{Ca}^{2+}$ transient amplitude was measured at the end of each tetani and expressed as $\left(F_{\max }-F_{0}\right) / F_{0}$ (Supplementary Fig. S2a). The integral of the $\mathrm{Ca}^{2+}$ transient decay following each tetanus was also calculated (Supplementary Fig. S2a). Average data were expressed as relative peak amplitudes and relative tail integrals to control for variability between cells.

Orai1 cloning and generation of dnOrai1 mice. Total RNA was extracted from mouse spleen using TRIZOL reagent according to the manufacturer's instructions. Before reverse transcription, RNA was treated with DNAase I for $15 \mathrm{~min}$ at room temperature. RNA was then reverse transcribed using Superscript III reverse transcriptase and oligo dTs. Murine WT Orail DNA was cloned using PCR and sequence-specific primers. A single amino-acid dominant-negative mutation in murine Orail (E108Q) was introduced using standard two-step site-directed mutagenesis. Three consecutive HA epitope (3xHA) sequences were similarly inserted in-frame at the extreme Orail C terminus. The dnOrail-3xHA construct was then cloned into a skeletal muscle-specific transgene vector containing a human troponin I enhancer, HSA (human skeletal muscle actin) promoter and a BGH (bovine growth hormone) polyadenylation signal (pcDNA3.1 $(+)$-hTnIHSA-BGHpA (gift from Dr Jeffery D. Molkentin) ${ }^{42}$. The vector was linearized and injected into pronuclei of fertilized mouse oocytes by the University of Rochester Medical Center transgenic core facility. Founder lines exhibiting different levels of transgene incorporation were generated in a mixed background (B6SJL hybrid). Line-5 mice, exhibiting medium level of muscle-specific dnOrail protein expression (Fig. 3), were then backcrossed into C57Bl6 background. Genotyping was performed by PCR using the following primers: forward: $5^{\prime}$-CAAGTCCAGCT CGACACAGA-3 ${ }^{\prime}$ reverse: $5^{\prime}$-CTGCATAGTCCGGGACGTCATAGG-3'.

Immunocytochemistry. Freshly isolated FDB fibres were fixed with $4 \%$ paraformaldehyde and labelled/co-labelled using different primary antibodies (see Supplementary Table S2 for details) followed by appropriate Rhodamine/Alexa Fluor 488-conjugated secondary antibodies. Rhodamine and Alexa 488 were sequentially excited using 543 and $488 \mathrm{~nm}$ lasers, and detected at 605/75 and 515/ $30 \mathrm{~nm}$, respectively, using a Nikon D-Eclipse C1 confocal microscope equipped with a $\times 40$, NA 1.3 oil-immersion objective. Images were averaged $(n=4)$ and deconvolved (two-dimensional blind) for presentation. probed using a mouse monoclonal anti-dystrophin antibody (MANDRA1, Abcam, 
1:500). Sections were visualized using Alexa Fluor 488 anti-mouse antibody and imaged as described above (in Immunocytochemistry). Fibre CSA was calculated using ImageJ software. Similar sections were alternatively stained using H\&E to assess muscle ultrastructure, specifically the presence of centrally nucleated fibres and fibrosis.

Electron microscopy. After killing, EDL and FDB muscles were fixed in situ at room temperature and maintained in fixative (3.5\% glutaraldehyde in $0.1 \mathrm{M}$ $\mathrm{NaCaCo}$ buffer, pH 7.2) until use. Small portions of muscle were post-fixed, embedded, stained en-block and sectioned ${ }^{43}$. Ultrathin sections $(\sim 50 \mathrm{~nm})$, after staining in $4 \%$ uranyl acetate and lead citrate, were examined with a Morgagni Series 268D electron microscope (FEI Company, Brno, Czech Republic), equipped with Megaview III digital camera.

Western blot analysis. TA muscles were excised and homogenized in ice-cold RIPA buffer and centrifuged at $4{ }^{\circ} \mathrm{C}, 16,000 \mathrm{~g}$ for $15 \mathrm{~min}$ and supernatants were collected for analysis. Prepared sample were solubilized in standard Laemmli sample buffer before electrophoresis. Proteins were separated by SDSpolyacrylamide gel electrophoresis, transferred to nitrocellulose membrane and immunostained with different primary antibodies followed by appropriate horseradish peroxidase-conjugated secondary antibodies (see Supplementary Table S2). Blots were visualized by enhanced chemiluminescence. Protein band densities were quantified using ImageJ software, using either GAPDH or $\alpha$-actinin as loading controls. Full-length images of immunoblots are shown in Supplementary Fig. S7.

Measurements of total SR $\mathbf{C a}^{2}{ }^{2}$. FDB fibres were loaded with $4 \mu \mathrm{M}$ fura-2FF $\mathrm{AM}$ at room temperature for $30 \mathrm{~min}$ followed by a 30 -min washout in dye-free Ringer's solution. Total SR $\mathrm{Ca}^{2+}$ content was determined by fura-2-FF fluorescence after local application of a $\mathrm{Ca}^{2+}$ release cocktail (ICE-10 $\mu \mathrm{M}$ ionomycin, $30 \mu \mathrm{M}$ cyclopiazonic acid and $100 \mu \mathrm{M}$ EGTA/ $\left./ \mathrm{Ca}^{2+}\right)^{33}$.

Myosin heavy chain analysis. TA, EDL, soleus and FDB muscles from 4-6month-old male and female WT and dnOrail mice were homogenized on ice in a buffer containing (in mM) 20 imidazole (pH 7.2), $150 \mathrm{KCl}$ and 0.2 EDTA with protease inhibitors. Tissue homogenates were centrifuged at $4{ }^{\circ} \mathrm{C}, 16,000 \mathrm{~g}$ for $15 \mathrm{~min}$. Pellets were resuspended in a buffer containing (in $\mathrm{mM}$ ) 50 Tris- $\mathrm{HCl}$ (pH 7.4), $150 \mathrm{NaCl}$ with protease inhibitors, snap frozen and stored at $80^{\circ} \mathrm{C}$ until use. Before analysis, prepared samples were diluted 1:1 in a sample buffer containing $0.16 \mathrm{M}$ Tris- $\mathrm{HCl}(\mathrm{pH} 6.8$ ), $40 \%$ glycerol, $4 \%$ SDS, $0.1 \mathrm{M}$ dithiothreitol and $0.02 \%$ bromophenol blue, and solubilized at $95^{\circ} \mathrm{C}$ for $5 \mathrm{~min}$. Myosin heavy chain isoforms were separated using SDS-polyacrylamide gel electrophoresis as described previously ${ }^{44,45}$ with modifications. Electrophoresis were carried out using a Bio-Rad Mini Protean system, with resolving gels containing $8 \%$ acrylamide/bis (99:1), $35 \%$ glycerol, $0.4 \%$ SDS, $0.2 \mathrm{M}$ Tris- $\mathrm{HCl}$ (pH 8.8), $0.1 \mathrm{M}$ glycine, $0.1 \%$ ammonium persulfate, $0.05 \% \mathrm{~N}, \mathrm{~N}, \mathrm{~N}^{\prime}, \mathrm{N}^{\prime}$-tetramethylethylenediamine and stacking gels containing $30 \%$ glycerol, $4 \%$ acrylamide/bis (49:1), $0.4 \%$ SDS, $70 \mathrm{mM}$ Tris-HCl (pH 6.7), $4 \mathrm{mM}$ EDTA, $0.1 \%$ ammonium persulfate and $0.05 \% \mathrm{~N}, \mathrm{~N}, \mathrm{~N}^{\prime}, \mathrm{N}^{\prime}-$ tetramethylethylenediamine. Protein $(1.5 \mu \mathrm{g})$ was loaded in each lane. Gels were electrophoresed at $140 \mathrm{~V}$ for $16-18 \mathrm{~h}$ at $4{ }^{\circ} \mathrm{C}$ in running buffers containing: outer chamber, $0.05 \mathrm{M}$ Tris, $0.075 \mathrm{M}$ glycine $0.05 \% \mathrm{SDS}$; inner chamber: eight times the concentration of outer chamber supplemented with $0.12 \% \beta$-mercaptoethanol. After electrophoresis, protein bands were visualized by silver staining, photographed, and relative composition of myosin heave chain isoforms were determined using ImageJ program.

In vitro muscle contraction assay. Muscle strength and fatigability were analysed in EDL muscles using an ASI muscle contraction system (Aurora Scientific), equipped with a 300C-LR dual mode force transducer and a 701C stimulator. Mice were anaesthetized by intraperitoneal injection of $100 \mathrm{mg} \mathrm{kg}^{-1}$ ketamine, $10 \mathrm{mg} \mathrm{kg}^{-1}$ xylazine and $2 \mathrm{mg} \mathrm{kg}^{-1}$ acepromazine. EDL muscles were exposed after removing TA muscles. The proximal and distal tendons of EDL muscle were then tied using 4-0 surgical suture, and EDL muscles were carefully excised and mounted between two platinum electrode plates while continuously perfused with oxygenated Ringer's solution in the experimental chamber ${ }^{46}$. Muscle optimal length $\left(L_{\mathrm{o}}\right)$ was determined using a series of $1 \mathrm{~Hz}$ stimulation and set at the length that generates the maximal force. Stimulus output was set at $120 \%$ of the voltage that elicits maximal force. Muscles were first equilibrated using three $500 \mathrm{~ms}$, $150 \mathrm{~Hz}$ tetani at $1 \mathrm{~min}$ intervals and then subjected to a force frequency, a single sustained high-frequency tetanus $(150 \mathrm{~Hz}, 2 \mathrm{~s})$ and/or a repetitive tetanic fatiguing (60 successive $50 \mathrm{~Hz}, 500 \mathrm{~ms}$ tetani) protocol. Muscle force was recorded using a Dynamic Muscle Control software and analysed using Clampfit 10.0 software. Muscle CSA and specific force were calculated as described previously ${ }^{46}$.

In vivo fatigue assays. Rotarod and treadmill running were used to assess the fatigability of 4-6-month-old male and female WT and dnOrail mice during rigorous exercise. For rotarod endurance task, mice were first acclimated to rotarod running for $1 \mathrm{~h}$ on a Rotamex-5 (Columbus Instruments) at 15 r.p.m. for two consecutive days. On the following day, mice were placed on the rotarod at an initial speed of 15 r.p.m. for $15 \mathrm{~min}$. The speed was then increased by 1 r.p.m. every $5 \mathrm{~min}$ for the following $30 \mathrm{~min}$ and then by 1 r.p.m. every $15 \mathrm{~min}$ for the rest of the 2-h task. In the event of a fall, the fall was noted and the mouse was returned to the rotarod. The number of cumulative falls over time during the task was recorded for each mouse. For treadmill (Columbus Instruments) experiments, mice were pre-trained for treadmill running 3 days before experiments, for 5 min each day at a speed of $5 \mathrm{~m} \mathrm{~min}^{-1}$ at a $15^{\circ}$ inclination. An acute exhaustion protocol was used for the treadmill task. After a 5 -min warm up at $5 \mathrm{~m} \mathrm{~min}^{-1}$ at a $15^{\circ}$ inclination, treadmill speed was increased $1 \mathrm{~m} \mathrm{~min}^{-1}$ every minute up to $20 \mathrm{~m} \mathrm{~min}^{-1}$ and remained at the maximum speed for an additional $40 \mathrm{~min}$. The entire task was $1 \mathrm{~km}$ in distance and $1 \mathrm{~h}$ in duration. Continued running was encouraged by delivering brief $(<1 s)$ spirits of air on the mouse's backside using a Whoosh Duster. The number of rests was recorded for each mouse. Finally, the number of cumulative falls (Rotarod) or cumulative rests (treadmill, binned every $5 \mathrm{~min}$ ) across multiple animals for each genotype were then averaged and plotted against the duration of task.

Data analysis. Results are expressed as mean \pm s.e.m. with statistical significance determined using Student's $t$-test for simple comparison and one-way analysis of variance followed by post hoc Student-Newman-Keuls test for multiple comparisons. $P<0.05$ were considered as statistically significant.

\section{References}

1. Parekh, A. B. On the activation mechanism of store-operated calcium channels. Pflugers Arch. 453, 303-311 (2006).

2. Liou, J. et al. STIM is a $\mathrm{Ca}^{2+}$ sensor essential for $\mathrm{Ca}^{2+}$-store-depletiontriggered $\mathrm{Ca}^{2+}$ influx. Curr. Biol. 15, 1235-1241 (2005).

3. Roos, J. et al. STIM1, an essential and conserved component of store-operated $\mathrm{Ca}^{2+}$ channel function. J. Cell Biol. 169, 435-445 (2005).

4. Zhang, S. L. et al. STIM1 is a $\mathrm{Ca}^{2+}$ sensor that activates CRAC channels and migrates from the $\mathrm{Ca}^{2+}$ store to the plasma membrane. Nature 437, 902-905 (2005).

5. Feske, S. et al. A mutation in Orail causes immune deficiency by abrogating CRAC channel function. Nature 441, 179-185 (2006).

6. Vig, M. et al. CRACM1 is a plasma membrane protein essential for storeoperated $\mathrm{Ca}^{2+}$ entry. Science 312, 1220-1223 (2006)

7. Feske, S. ORAI1 and STIM1 deficiency in human and mice: roles of storeoperated $\mathrm{Ca}^{2+}$ entry in the immune system and beyond. Immunol. Rev. 231, 189-209 (2009).

8. Kurosaki, T. \& Baba, Y. $\mathrm{Ca}^{2+}$ signaling and STIM1. Prog. Biophys. Mol. Biol. 103, 51-58 (2010).

9. Parekh, A. B. \& Putney, Jr. J. W. Store-operated calcium channels. Physiol. Rev. 85, 757-810 (2005).

10. Smyth, J. T. et al. Activation and regulation of store-operated calcium entry. J. Cell Mol. Med. 14, 2337-2349 (2010).

11. $\mathrm{Li}$, T. et al. STIM1-Ca ${ }^{2+}$ signaling is required for the hypertrophic growth of skeletal muscle in mice. Mol. Cell Biol. 32, 3009-3017 (2012).

12. Lyfenko, A. D. \& Dirksen, R. T. Differential dependence of store-operated and excitation-coupled $\mathrm{Ca}^{2+}$ entry in skeletal muscle on STIM1 and Orail. J. Physiol. 586, 4815-4824 (2008).

13. Stiber, J. et al. STIM1 signalling controls store-operated calcium entry required for development and contractile function in skeletal muscle. Nat. Cell Biol. 10, 688-697 (2008).

14. Yarotskyy, V. \& Dirksen, R. T. Temperature and RyR1 regulate the activation rate of store-operated $\mathrm{Ca}^{2+}$ entry current in myotubes. Biophys. J. 103, 202-211 (2012).

15. Darbellay, B., Arnaudeau, S., Bader, C. R., Konig, S. \& Bernheim, L. STIM1L is a new actin-binding splice variant involved in fast repetitive $\mathrm{Ca}^{2+}$ release. J. Cell Biol. 194, 335-346 (2011).

16. Prakriya, M. \& Lewis, R. S. Potentiation and inhibition of $\mathrm{Ca}^{2+}$ releaseactivated $\mathrm{Ca}^{2+}$ channels by 2 -aminoethyldiphenyl borate (2-APB) occurs independently of IP(3) receptors. J. Physiol. 536, 3-19 (2001).

17. Prakriya, M. \& Lewis, R. S. Separation and characterization of currents through store-operated CRAC channels and $\mathrm{Mg}^{2+}$-inhibited cation (MIC) channels. J. Gen. Physiol. 119, 487-507 (2002).

18. Kurebayashi, N. \& Ogawa, Y. Depletion of $\mathrm{Ca}^{2+}$ in the sarcoplasmic reticulum stimulates $\mathrm{Ca}^{2+}$ entry into mouse skeletal muscle fibres. J. Physiol. 533, 185-199 (2001).

19. Pan, Z. et al. Dysfunction of store-operated calcium channel in muscle cells lacking mg29. Nat. Cell Biol. 4, 379-383 (2002).

20. Zhao, X. et al. Enhanced resistance to fatigue and altered calcium handling properties of sarcalumenin knockout mice. Physiol. Genomics 23, 72-78 (2005)

21. Rosenberg, P. et al. TRPC3 channels confer cellular memory of recent neuromuscular activity. Proc. Natl Acad. Sci. USA 101, 9387-9392 (2004).

22. Darbellay, B. et al. STIM1- and Orai1-dependent store-operated calcium entry regulates human myoblast differentiation. J. Biol. Chem. 284, 5370-5380 (2009). 
23. Soboloff, J., Rothberg, B. S., Madesh, M. \& Gill, D. L. STIM proteins: dynamic calcium signal transducers. Nat. Rev. Mol. Cell Biol. 13, 549-565 (2012).

24. Edwards, J. N. et al. Ultra-rapid activation and deactivation of store-operated $\mathrm{Ca}^{2+}$ entry in skeletal muscle. Cell Calcium 47, 458-467 (2010).

25. Launikonis, B. S., Murphy, R. M. \& Edwards, J. N. Toward the roles of store-operated $\mathrm{Ca}^{2+}$ entry in skeletal muscle. Pflugers Arch. 460, 813-823 (2010).

26. Launikonis, B. S. \& Ríos, E. Store-operated $\mathrm{Ca}^{2+}$ entry during intracellular $\mathrm{Ca}^{2+}$ release in mammalian skeletal muscle. J. Physiol. 583, 81-97 (2007).

27. Dirksen, R. T. Checking your SOCCs and feet: the molecular mechanisms of $\mathrm{Ca}^{2+}$ entry in skeletal muscle. J. Physiol. 587, 3139-3147 (2009).

28. Kerppola, T. K. Design and implementation of bimolecular fluorescence complementation (BiFC) assays for the visualization of protein interactions in living cells. Nat. Protoc. 1, 1278-1286 (2006).

29. Kerppola, T. K. Complementary methods for studies of protein interactions in living cells. Nat. Methods 3, 969-971 (2006).

30. Kerppola, T. K. Visualization of molecular interactions by fluorescence complementation. Nat. Rev. Mol. Cell Biol. 7, 449-456 (2006)

31. Canato, M. et al. Massive alterations of sarcoplasmic reticulum free calcium in skeletal muscle fibers lacking calsequestrin revealed by a genetically encoded probe. Proc. Natl Acad. Sci. USA 107, 22326-22331 (2010).

32. Capote, J., Bolanos, P., Schuhmeier, R. P., Melzer, W. \& Caputo, C. Calcium transients in developing mouse skeletal muscle fibres. J. Physiol. 564, 451-464 (2005).

33. Loy, R. E. et al. Muscle weakness in Ryr1I4895T/WT knock-in mice as a result of reduced ryanodine receptor $\mathrm{Ca}^{2}+$ ion permeation and release from the sarcoplasmic reticulum. J. Gen. Physiol. 137, 43-57 (2011).

34. Cheng, K. T., Liu, X., Ong, H. L. \& Ambudkar, I. S. Functional requirement for Orail in store-operated TRPC1-STIM1 channels. J. Biol. Chem. 283, 12935-12940 (2008).

35. Zhao, X., Moloughney, J. G., Zhang, S., Komazaki, S. \& Weisleder, N. Orai1 mediates exacerbated $\mathrm{Ca}^{2+}$ entry in dystrophic skeletal muscle. PLoS One 7, e49862 (2012)

36. Edwards, J. N. et al. Upregulation of store-operated $\mathrm{Ca}^{2+}$ entry in dystrophic mdx mouse muscle. Am. J. Physiol. Cell Physiol. 299, C42-C50 (2010).

37. Cully, T. R. \& Launikonis, B. S. Store-operated $\mathrm{Ca}^{2+}$ entry is not required for store refilling in skeletal muscle. Clin. Exp. Pharmacol. Physiol. 40, 338-344 (2013).

38. Thornton, A. M. et al. Store-Operated $\mathrm{Ca}^{2+}$ Entry (SOCE) Contributes to Normal Skeletal Muscle Contractility in young but not in aged skeletal muscle. Aging 3, 621-634 (2011).

39. Duke, A. M., Hopkins, P. M., Calaghan, S. C., Halsall, J. P. \& Steele, D. S. Store-operated $\mathrm{Ca}^{2+}$ entry in malignant hyperthermia-susceptible human skeletal muscle. J. Biol. Chem. 285, 25645-25653 (2010).

40. Wei, L. et al. Mitochondrial superoxide flashes: metabolic biomarkers of skeletal muscle activity and disease. FASEB J. 25, 3068-3078 (2011).
41. Rossi, A. E., Boncompagni, S., Wei, L., Protasi, F. \& Dirksen, R. T. Differential impact of mitochondrial positioning on mitochondrial $\mathrm{Ca}^{2+}$ uptake and $\mathrm{Ca}^{2+}$ spark suppression in skeletal muscle. Am. J. Physiol. Cell Physiol. 301, C1128-C1139 (2011).

42. Brennan, K. J. \& Hardeman, E. C. Quantitative analysis of the human alphaskeletal actin gene in transgenic mice. J. Biol. Chem. 268, 719-725 (1993).

43. Boncompagni, S. et al. Characterization and temporal development of cores in a mouse model of malignant hyperthermia. Proc. Natl Acad. Sci. USA 106, 21996-22001 (2009)

44. Talmadge, R. J. \& Roy, R. R. Electrophoretic separation of rat skeletal muscle myosin heavy-chain isoforms. J. Appl. Physiol. 75, 2337-2340 (1993).

45. Mizunoya, W., Wakamatsu, J.-i., Tatsumi, R. \& Ikeuchi, Y. Protocol for highresolution separation of rodent myosin heavy chain isoforms in a mini-gel electrophoresis system. Anal. Biochem. 377, 111-113 (2008).

46. Hakim, C., Li, D. \& Duan, D. Monitoring murine skeletal muscle function for muscle gene therapy. in Muscle Gene Therapy. Vol 709 (ed. Duan, D.) 75-89 (Humana Press, 2011).

\section{Acknowledgements}

We thank Dr. Jeffery Molkentin for kindly providing the muscle-specific transgene vector used to generate the dnOrail mice, Dr. Veit Flockerzi for providing an anti-Orail antibody, Ryan Loy for help with video editing, and Linda Groom and Sara Geitner for providing excellent technical assistance. We also thank Dr. Thurman Wheeler for providing access to the mouse treadmill used in this study. This study was supported by an NIH Grant (AR059646 to R.T.D.), the Academia Dei Lincea Fund (to L.W.L.) and a NSF Predoctoral Fellowship (to E.M.C.)

\section{Author contributions}

R.T.D. and L.W.L. designed research; S.B., E.M.C. and L.W.L. performed experiments and analysed data; and R.T.D., L.W.L. and F.P. wrote the manuscript.

\section{Additional information}

Supplementary Information accompanies this paper at http://www.nature.com/ naturecommunications

Competing financial interests: The authors declare no competing financial interests.

Reprints and permission information is available online at http://npg.nature.com/ reprintsandpermissions/

How to cite this article: Wei-LaPierre, L. et al. Orail-dependent calcium entry promotes skeletal muscle growth and limits fatigue. Nat. Commun. 4:2805 doi: 10.1038/ncomms3805 (2013). 